\title{
Desarrollo de material didáctico multimedia del cultivo de tejidos vegetales con aplicaciones agrarias y ambientales
}

\section{Development of multimedia teaching material of plant tissue culture with agricultural and environmental applications}

\author{
Desenvolvimento de material de ensino multimídia de \\ cultura de tecidos de plantas com aplicações agrícolas \\ e ambientais.
}

\author{
Oscar Agudelo Varela ${ }^{1}$, Miguel Macgayver Bonilla Morales ${ }^{2} \&$ Adriana Carolina Aguirre Morales $^{3}$ \\ ${ }^{1}$ Ingeniero de Sistemas, Magister en Ciencias de la Información y la Comunicación.
}

${ }^{2}$ Lic. Pdn. Agrop., Magister en Ciencias Biológicas. ${ }^{3}$ Ingeniera Agrónoma, Magister en Ciencias Biológicas.

${ }^{1}$ G. I. Horizonte Mediático, Universidad de los Llanos, Sede Barcelona. Villavicencio, Colombia. ${ }^{2,3}$ CorpOrquídea y G.I. EduCiTec, Universidad de los Llanos, Sede Barcelona. Villavicencio, Colombia.

10scar.agudelo@unillanos.edu.co, ${ }^{2}$ mmbonillam@unal.edu.co, ${ }^{3}$ acaguirrem@gmail.com

\section{Resumen}

Las aplicaciones pedagógicas tradicionales se fundamentan en el desarrollo de la clase con marcador y tablero, y con leves avances en el manejo de diapositivas; sin embargo, las tecnologías digitales presentan una serie de novedades que complementan el trabajo de la enseñanza con aprendizaje autónomo, que puede desarrollar el educando en áreas agrarias y ambientales. El desarrollo de material didáctico multimedia MDM complementa las actividades curriculares de los cursos, particularmente, los que tienen laboratorio, y por otro lado, generan en el estudiante habilidades para el aprendizaje autónomo. Se desarrolló un MDM para el aprendizaje de las prácticas realizadas en el laboratorio de cultivos vegetales, con arquitectura cliente/ servidor, usando tecnologías actuales en el desarrollo de software, con características de tiempo real y bajo el paradigma de desarrollo de aplicaciones isomórficas. Con base al MDM, se realizó evaluación cualitativa por parte de estudiantes de Licenciatura en Producción Agropecuaria, la cual reporto un $86 \%$ de aprobación del material. Se encontró que las herramientas digitales para el aprendizaje, facilitan y mejoran en cierta medida el aprendizaje de los educandos. El uso de tecnologías nuevas como React, permite mejorar el renderizado de las páginas y optimiza la carga del servidor en las peticiones necesarias por parte del aprendiz, y hace más eficiente el engranaje de enseñanza-aprendizaje. De esta manera, el MDM permitirá fundamentar el desarrollo de habilidades y destrezas en la producción agraria y en el manejo de especies de ecosistemas con ambientes naturales.

Palabras clave: aprendizaje virtual, educación, laboratorio virtual, tecnologías en la educación. 


\section{Abstract}

Traditional pedagogical applications are based on the development of the class with scoreboard and board, and with slight advances in the handling of slides; however, the digital technologies present a series of innovations that complement the work of the teaching with autonomous learning, that can develop the educating in agrarian and environmental areas. The development of multimedia didactic material MDM complements the curricular activities of the courses, particularly those that have a laboratory, and on the other hand, generate in the student skills for autonomous learning. An MDM was developed for the learning of the practices carried out in the plant culture laboratory, with client/server architecture, using current technologies in software development, with realtime characteristics and under the paradigm of Development of isomorphic applications. Based on MDM, qualitative evaluation was carried out by undergraduate students in agricultural production, which reported $86 \%$ approval of the material. Digital learning tools were found to facilitate and improve learning of learners to some extent. The use of new technologies like React, allows to improve the rendering of the pages and optimizes the load of the server in the necessary requests by the Apprentice, and makes more efficient the teaching-learning gear. In this way, MDM will support the development of skills and skills in agrarian production and in the management of ecosystem species with natural environments.

Key-words: virtual learning, education, virtual lab, technologies in education.

\section{Resumen}

As aplicações pedagógicas tradicionais são baseadas no desenvolvimento da classe com marcador e placa, e com ligeiros avanços na manipulação de slides; no entanto, as tecnologias digitais apresentam uma série de novidades que complementam o trabalho de ensino com aprendizagem autônoma que pode desenvolver o aluno em áreas agrícolas e ambientais. O desenvolvimento de material didáctico multimídia MDM complementa as atividades curriculares dos cursos, particularmente aqueles que possuem um laboratório, e, por outro lado, geram habilidades estudantis para a aprendizagem autônoma. Um MDM foi desenvolvido para aprender as práticas realizadas no laboratório de cultivo de plantas, com arquitetura cliente / servidor, usando tecnologias atuais em desenvolvimento de software, com características em tempo real e sob o paradigma do desenvolvimento de aplicativos isomórficos. Com base no MDM, uma avaliação qualitativa foi realizada por estudantes de graduação em Produção Agrícola, que relataram uma aprovação de $86 \%$ do material. Verificou-se que as ferramentas de aprendizagem digital facilitam e melhoram, até certo ponto, a aprendizagem dos alunos. O uso de novas tecnologias, como Reagir, permite melhorar a renderização das páginas e otimizar a carga do servidor nas solicitações necessárias por parte do aprendiz e torna a ferramenta de ensinoaprendizagem mais eficiente. Desta forma, o MDM permitirá basear o desenvolvimento de habilidades e habilidades na produção agrícola e na gestão de espécies de ecossistemas com ambientes naturais.

Palavras chave: aprendizagem virtual, educação, laboratório virtual, tecnologias na educação.

\section{Introducción}

Las tecnologías en la educación -TE- fomentan la cultura del uso de las herramientas en sistemas con fines más allá de lo informático, pues generan ambientes virtuales de aprendizaje que fortalecen la enseñanza y aprendizaje del usuario o educando (Guzmán, 2006). De esta manera, el uso de software aplicativos se han sumado Instituciones Educativas y Universidades de Norteamérica (Carnoy, 2004), Centroamérica (Romero et al., 2010) y Suramérica (Varela et al., 2014, 2015) obteniendo resultados satisfactorios de aprendizaje por parte de los educando. No obstante, 
el sistema educativo en Colombia, generalmente, no utiliza las tecnologías de la innovación y la comunicación (TICs) para potenciar la calidad de la enseñanza y aprendizaje, que se presenta cuando se trabaja en conjunto con las TE (Carnoy, 2004).

Dentro de las ciencias aplicadas que particularmente requieren para su accionar las ciencias básicas, se encuentra el área de Biotecnología que ha tenido un enfoque en el cultivo de tejidos vegetales (CTV) para que los profesionales de diferentes áreas desarrollen habilidades y destrezas técnicas para la solución de problemas mediante la investigación y las empresas (Orozco \& Olaya, 2016). En este sentido, el CTV posee una amplia incidencia en las aplicaciones agrícolas como la generación de plantas libres de patógenos, masivamente, a bajo costo, entre otras alterativas que permiten mejorar el agro en Colombia, aunque, también tiene diversas aplicaciones biológicas, químicas como otros ejemplos asociados a la agroindustria (Gonzáles et al., 2010). Además, esté tópico para su desarrollo requiere de laboratorios específicos para cada una de las actividades que implican mientras los explantes entran a la etapa in vitro, luego ex vitro, y finalmente, campo (Hernández, 2008; Ramírez et al., 2012). Por su parte, también se incluye en el programa de manejo y conservación de la flora asociado a especies nativas y endémicas mediante la generación de bancos de germoplasma ex situ, y adicionalmente, de este material, se utiliza para la repoblación en ecosistemas con ambientes naturales.

Por tal motivo, los estudiantes no poseen materiales multimedia que les permita generar aprendizaje autónomo, independiente del profesor, y que relacione la realidad de las habilidades técnicas necesarias para el desarrollo de labores en CTV. En este orden lógico, se realizó el diseño y desarrollo un laboratorio virtual de CTV con énfasis para promover el aprendizaje en componentes de biotecnología (CTV) en estudiantes universitarios, además de servir de apoyo para los docentes en sus clases.

\section{Materiales y métodos}

Para el desarrollo del laboratorio virtual de CTV se aplicó la Metodología Ingeniería de Software Educativo (MelSE) propuesta por (Abud, 2009), con la finalidad de que los usuarios (educadores y educandos) puedan alcanzar aprendizaje autónomo. Esta metodología contiene las siguientes fases: análisis, diseño, desarrollo y prueba piloto.

\section{Análisis}

Se realizó una entrevista a los estudiantes de Ingeniería Agronómica (IA) y de la Licenciatura en Producción Agropecuaria (LPA) de la línea de profundización en Biotecnología y Propagación Vegetal que pertenecen a VII y VIII semestre, respectivamente. Las preguntas relacionaron: qué temas fueron los difíciles, qué material didáctico les hubiera gusta utilizar y qué aspectos mejorarían del curso. Dados los problemas en el aprendizaje teórico-práctico, ya que no manejan los conceptos adecuados sobre la temática ni las labores aplicativas que se ejecutan en el laboratorio (Levitus, 2010). Se revisó el material didáctico que hay sobre el desarrollo de las temáticas en laboratorios de CTV. Como el objetivo del análisis es determinar el contexto en el cual se va a usar el laboratorio virtual se tendrán los siguientes indicadores en esta fase: población objetivo y sus características; temas del contenido y sus características, principios pedagógicos aplicables -Autoregulación del aprendizaje-, y modos de uso de la aplicación.

\section{Diseño}

Diseño Educativo. Esta fase se enfoca en las temáticas que hacen relación al cultivo de tejidos vegetales de acuerdo a los temas planteadas en los textos de Levitus et al. (2010) y Roca \& Mroinski (1991), además de los exigidos por la malla curricular de los programas en cuestión. A continuación se listan los temas seleccionados por los docentes de la línea de profundización en CTV: i) conociendo el laboratorio, ii) preparación de stock, iii) preparación de Medios de Cultivo, iv) micropropagación in vitro, v) etapa ex vitro, vi) cultivo in vitro de meristemos y ápices y vii) rescate y cultivo de embriones cigóticos. 
Diseño Comunicativo y Computacional. El laboratorio virtual de CTV se desarrolló en un lenguaje para web, con la finalidad de que exista mayor disponibilidad y acceso a cualquier hora y lugar mediante cualquier dispositivo digital. Para lo cual se definió la estructura global y las configuraciones de diseño y plantillas para el despliegue de la información. Lo anterior implica, el diseño gráfico, la colocación de íconos, la definición del texto descriptivo en pantalla, la especificación y títulos de las ventanas, y las especificaciones de los elementos principales y secundarios del menú. Se adaptó una estructura jerárquica con lo que se logra un control de los contenidos, clasificándolos cada uno por módulos. Una vez establecida la estructura del laboratorio virtual de CTV, e identificados los componentes -páginas, guiones, animaciones y otras funciones de proceso-, Se definen las rutas de navegación que permitan al estudiante acceder al contenido y a los servicios del laboratorio virtual de CTV (Figura 1).

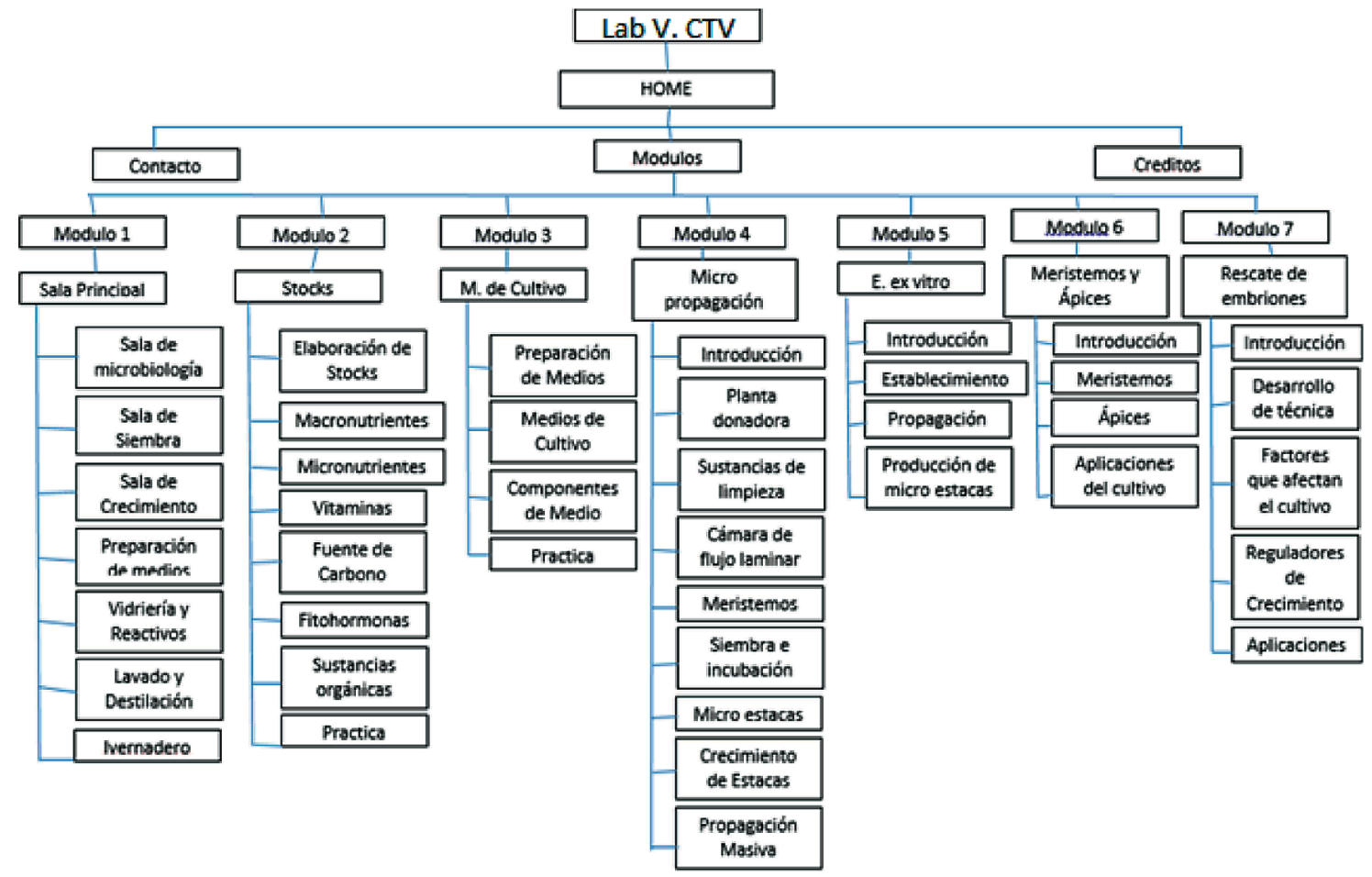

Figura 1. Mapa de navegación detallado sobre el MDM en CTV.

\section{Desarrollo}

El laboratorio virtual se desarrolló bajo la arquitectura cliente servidor e implementando el paradigma de desarrollo de aplicaciones isomórficas. El contenido de los temas está compuesto por texto, imágenes animaciones y simulaciones. Los cuales son planteados por los docentes o expertos en CTV, los encargados de digitalizar los textos son los monitores, los programadores toman los esbozos de las animaciones y simulaciones para su implementación en HTML5 o CSS3, las cuales son seleccionadas teniendo en cuenta los temas de difícil comprensión para los estudiantes y que aporte claridad a los conceptos, el diseñador gráfico genera las imágenes y retoca las fotografías, todos esos elementos son enviados al integrador, que se encarga de colocarlos en la plantilla. Las librerías y lenguajes utilizados para el desarrollo del laboratorio virtual, son libres y fueron las siguientes: i) Bootstrap, ii) ECMAScript 6, iii) React.js, iv) NodeJS, v) JQuery, vi) JSX, vii) CSS3, viii) HTML5. En esta fase se realizaron siete iteraciones, una por cada tema establecido, a cada una de ellas se les realizo su respectiva fase de despliegue. 
La Figura 2 corresponde a las interfaces de pantalla principal, módulo preparación de stock y prácticas de laboratorio, en las cuales se pueden apreciar colores e imágenes atractivas y vibrantes para los estudiantes, que permite generar una empatía con la aplicación. La Figura 2A presenta los siete módulos con imágenes que las identifican, la Figura 2B presenta los distintos elementos que se tienen en cuenta para los stocks, y, finalmente, la Figura 2C es la plantilla de las prácticas de laboratorio.

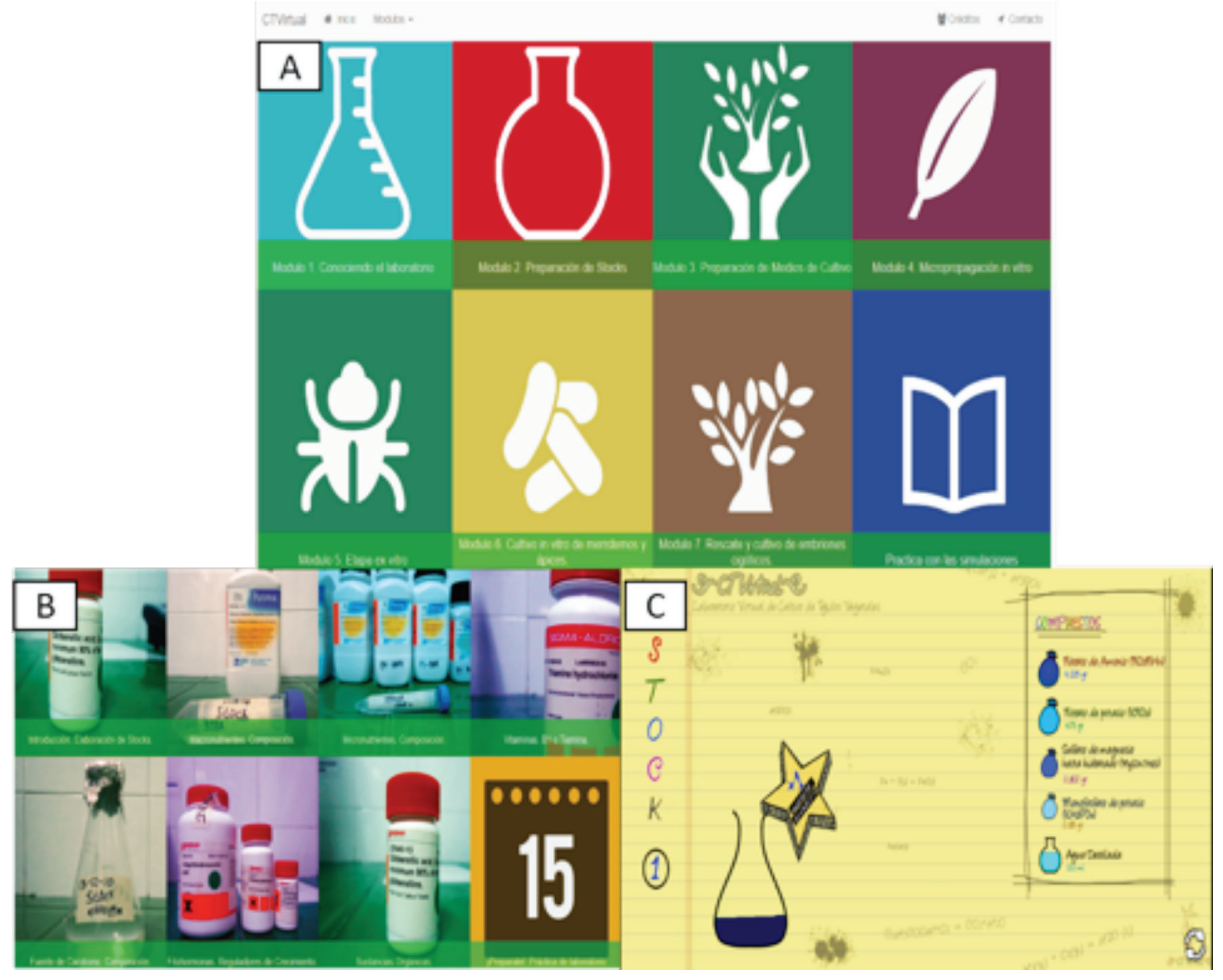

Figura 2. MDM en CTV. A) Pantalla o interfaz principal, B) Módulo preparación de stock y, C) practica de laboratorio.

\section{Fase de Pruebas}

Después de haber generado el aplicativo web, el software debe ser probado para descubrir el máximo de errores posibles antes de su entrega a los estudiantes y docentes. De acuerdo a esto, se diseñó una serie de prueba del software donde se tuvieron en cuenta las siguientes estrategias: a) Se verifico la navegación de cada módulo construido, en búsqueda de errores, estas verificaciones las realizaron estudiantes de X semestre de Ingeniería de Sistemas; b) Se realizaron pruebas de aceptación con los docentes y algunos estudiantes de LPA. Estas se hicieron independientemente a cada módulo de la aplicación.

\section{Prueba piloto}

Esta prueba se realizó con 12 estudiantes del curso de tejidos vegetales -formalmente Propagación
Vegetal- de séptimo semestre del programa de LPA, los cuales utilizaron el laboratorio virtual y posteriormente se recolecto información, mediante la aplicación de un cuestionario que indago sobre las características de presentación de la información, aspectos de la tecnología y aspectos didácticos del laboratorio virtual de CTV. Los resultados de la prueba piloto se utilizaron para realizar ajustes con miras a la aplicación de la prueba de campo. El cuestionario se realizó según valoración en la escala de Likert, así: totalmente de acuerdo $=4$; de acuerdo $=3$; en desacuerdo $=2$; y totalmente en desacuerdo $=1$ (Pérez, 2004).

Algunas de las preguntas en el aspecto del contenido fueron: La estructura del material permite la consulta de las prácticas y sus contenidos, los contenidos se relacionan con los temas de laboratorio 
de CTV, la información permite interacción fácil con el usuario y los contenidos presentan animaciones y simulaciones. En el componente de tecnología: facilidad de instalación y uso del software. La interfaz es dinámica y agradable, el diseño gráfico está relacionado y es adecuado al contenido, el diseño técnico de animaciones y simulaciones es adecuada y el sistema de navegación es apropiado. Los aspectos del componente de didáctica son: los objetivos de aprendizaje están definidos, los objetivos de aprendizaje se relacionan con los contenidos y favorece el autoaprendizaje del cultivo de tejidos vegetales.

Para lograr una mayor aceptación por parte de los estudiantes, uno de los aspectos a tener en cuenta es la usabilidad y un factor importante es la velocidad de carga y respuesta de la aplicación (Hernández, 2016), con el fin de establecer esta medida se realizaron pruebas con 2 herramientas de uso web y con opciones de uso libre como lo son Pingdom y PageSpeed Insights. El análisis realizado con Pingdom estableció que el $61 \%$ de la carga de los 3,5 MB, corresponde a es Script y el $30 \%$ a imágenes, el tiempo de carga está entre los parámetros considerados de carga normal (Hernández, 2016), otros indicadores se pueden ver en la Figura 3.
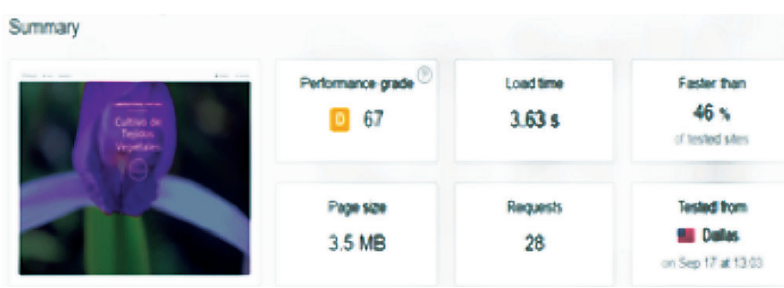

Figura 3. Pingdom, resumen de análisis para el MDM en CTV.

Page Speed Insights es una herramienta ofrecida por Google a los desarrolladores, la cual realiza un análisis a la página web y genera una evaluación de 0 a 100, siendo 100 la mejor evaluación, adicionalmente brinda sugerencia para mejorar la velocidad de carga y de despliegue en móviles. La evaluación en modo pc arrojo el resultado de 20 y sugerencias a elementos que deben corregirse, tales como: i) habilitar compresión, ii) Minificar JavaScript, iii) Optimizar imágenes, iv) Habilitar compresión. En la pestaña de móvil, PageSpeed Insights arrojo el resultado de 19 (ver Figura 4), además de los elementos que deben corregirse expuesto anteriormente, recomienda algunos que podrían corregirse: i) Prioriza el contenido visible, ii) Especificar caché de navegador, iii) Minificar CSS, iv) Minificar HTML.

\section{hetp://190.60.95.25:3000/m/ \\ Móvil Ordenador \\ $20 / 100$ Resumen de sugerenciat}

\section{$19 / 100$ Velocidad}

Figura 4. Page Speed Insights, resumen de análisis relacionados con el MDM en CTV.

Adicionalmente Page Speed Insights ofrece un puntaje sobre la Experiencia de usuario, en la cual el laboratorio virtual obtuvo 100 de 100, ese puntaje se basó en los siguientes elementos: i) adaptación del contenido a la ventana gráfica, ii) aplicar el tamaño adecuado a los botones táctiles, son lo suficientemente grandes para que los usuarios los puedan tocar en una pantalla táctil, iii) tu página especifica una ventana gráfica que se adapta al tamaño del dispositivo, lo que permite que se procese correctamente en todos los dispositivos, iv) evitar los plugins, Parece que tu página no utiliza plugins y esto podría impedir que el contenido se utilice en muchas plataformas, v) Utilizar tamaños de fuente que se puedan leer.

\section{Resultados y discusión}

Se generó un software educativo que permite a los interesados, educadores y educandos, adquirir conocimientos y destrezas en el área de laboratorio de cultivo de tejidos vegetales. El desarrollo de este objeto virtual de aprendizaje tiene las propiedades específicas para que el aprendiz mediante la interacción pueda ir captando los saberes necesarios para su manejo y simultáneamente, comprenda lo 
relacionado con la temática. Dentro de las experiencias relacionadas con el desarrollo de MDM en las ciencias se encuentra el realizado en morfología vegetal (Varela et al., 2014) e histología vegetal (Varela et al., 2015) que también permiten que el usuario pueda mediante la interfaz, la diagramación, el diseño, las pautas pedagógicas y metodológicas para el manejo de conceptos y prácticas puedan alcanzar habilidades metacognitivas para la comprensión de un saber específico.

EI MDM en CTV se fundamentó en los saberes básicos y aplicados en esta área de la biotecnología, siguiendo los principios del conocimiento del laboratorio, preparación de stocks, medios de cultivo y técnicas de micropropagación de material vegetal. De igual manera, Ramírez et al. (2012) registran estos tópicos como los centrales en la enseñanza y aprendizaje, ya que son el sustento para desarrollar habilidades cognitivas y técnicas en el CTV. Además, estos temas que se plantea el Laboratorio Virtual en CTV tienen como eje como lineamentos textos de vital importancia en el aprendizaje de los procedimientos y técnicas en el CTV como lo establecido en el texto clásico de Roca et al. (1991) u otro más actualizado como el de Levitus et al. (2010).

Dentro de las temáticas desarrolladas en la TE la micropropagación in vitro es un ítem complejo porque implica destreza y habilidad, pero el Laboratorio virtual de CTV incluye aplicaciones teóricas que fundamentan los conocimientos previos como la visualización de los procesos para la claridad en la aplicación de forma didáctica. La prioridad en los perfiles profesionales de las carreras que desarrollan competencias en la biotecnología, lo primordial es que el profesional tenga los componentes conceptuales y consecutivamente las destrezas manuales para que mediante las aplicaciones pueda producir material vegetal (Gonzáles et al., 2010; Orozco \& Olaya, 2016).

La interacción virtual implica profundizar los conocimientos teóricos de base con un alcance desde lo virtual, con la intención de alcanzar una experiencia significativa que lleve al educador o educando a percibir la realidad real, de tal manera, sirva como modelo para su imitación en el laboratorio o área de trabajo. En este orden de ideas, lo definido por Abud (2009) redondea de manera clara la columna vertebral del CTV siendo eje de un modelos simulador de la realidad con base lo establecido por Vidal \& González (2002) que finalmente permiten generar una cultura científica para la resolución de problemas de cualquier índole que se desarrollen en laboratorio (Reigosa \& Jiménez, 2000), como los que acomete el CTV.

\section{Conclusiones}

Con la aplicación de nuevas y actualizadas tecnologías usadas para el desarrollo de software con perspectiva a la construcción de material didáctico multimedia, se mejora la usabilidad, y por añadidura la experiencia del usuario, educador y educando, permitiendo el aprendizaje de los interesados, particularmente, en el área de cultivo de tejidos vegetales que requiere conceptos claros y habilidades técnicas específicas. Además, la sincronización de las animaciones, simulaciones y contenidos digitales, sumando la disponibilidad en la web, permite ampliar el acceso a las temáticas en estudio y a mejorar el rendimiento de los estudiantes en CTV con énfasis en la producción agraria y su desarrollo sostenible y sustentable con el ambiente, particularmente, ahondado el manejo y conservación de especies endémicas y nativas.

\section{Agradecimientos}

Los autores expresan sus agradecimientos a la Dirección General de Investigaciones de la Universidad de los Llanos por su apoyo y financiación del proyecto "Desarrollo de laboratorio virtual de cultivo de tejidos vegetales, utilizando una estrategia metacognitiva para mejorar el aprendizaje de los estudiantes de Ingeniería Agronómica y Licenciatura en Producción Agropecuaria de la Universidad de los Llanos". 


\section{Literatura citada}

1. Abud, A. (2009). Me ISE: Metodología de Ingeniería de software educativo. Revista internacional de educación en ingeniería. 2 (1), 1-9.

2. Carnoy, M. (2004). Las TIC en la enseñanza: posibilidades y retos. UOC.19 p. Recuperado de: $h t t p: / / w w w$. uoc.edu/inaugural04/esp/carnoy1004.pdf

3. Gonzales, C., Villa, J. \& Bravo J. (2010). La biotecnología como empresa". Unicauca. 8(1), 83-92.

4. Guzmán, J. (2006). Las TIC y las crisis de la educación: algunas claves para su comprensión. Recuperado de: http://www.virtualeduca.org/documentos/yanez.pdf.

5. Hernández, D. (2016). Cómo medir optimizar velocidad página web. Recuperado de: http://alfredohernandezdiaz.com/2015/09/29/comomedir-optimizar-velocidad-pagina-web/

6. Hernández, M. (2008). Propuesta de apoyo para la gestión eficiente de la biotecnología. Revista EAN. 62, 5-26.

7. Levitus, G., Echenique, V., Rubistein, C., Hopp, E. \& Mroginski, L. (2010). Biotecnología y mejoramiento vegetal II. ARGENBIO (Consejo Argentino para la Información y el Desarrollo de la Biotecnología). Ediciones: Instituto Nacional de Tecnología Agropecuaria. 648 p.

8. Orozco, L. \& Olaya, D. (2016). Indicadores del programa nacional de biotecnología. Observatorio Colombiano de Ciencia y Tecnología. 23 p. Recuperado de: https://es.scribd.com/document/3347516/ProgramaNacional-de-Biotecnologia-Indicadores

9. Pérez, G. (2004). Investigación cualitativa. Retos e interrogantes. 2 Vol. Ed. La Muralla Madrid. 72 p.
10. Ramírez, H., Guevera, M. \& Escobar, R. (2012). Cultivo de Tejidos Vegetales conceptos y prácticas. Guía de estudio. Universidad Nacional de Colombia sede Palmira. Palmira, Valle de Cauca, Colombia. 227p.

11. Reigosa, E. \& Jiménez, P. (2000). La cultura científica en la resolución de problemas en el laboratorio. Enseñanza de las ciencias. 18(2), 275-284.

12. Roca, W. \& Mroginski, L. (1991). Cultivo de tejidos en la Agricultura. Fundamentos y aplicación. Centro de Investigación en Agricultura Tropical, Cali-Colombia. 969 p.

13. Romero, P., Domínguez, J. \& Guillermo, C. (2010). EI uso de las Tic's en la educación de comunidades rurales y urbanas del sureste de México. Revista de Educación a Distancia. 22,1-19.

14. Varela, A., Bonilla, M. \& Romero, A. (2014). Desarrollo de material multimedia en Morfología Vegetal y su implementación utilizando una estrategia metacognitiva para fortalecer el aprendizaje de los estudiantes de Licenciatura en Producción Agropecuaria, Universidad de los Llanos. In Twelfth LACCEI Latin American and Caribbean Conference for Engineering and Technology. 1-9.

15. Varela, A., Bonilla, M. \& Romero, A. 2015. Implementación de Material Didáctico Multimedia en Histología Vegetal Mediante una Estrategia Metacognitiva para Fortalecer el Aprendizaje en los Estudiantes de I, II y IV Semestres de la Licenciatura en Producción Agropecuaria, Universidad de los Llanos. In 13th LACCEI Annual International Conference. 1-5.

16. Vidal, G. \& González, H. (2002). Evaluación pedagógica del simulador del laboratorio químico modelchemlab. Revista Pedagogía Universitaria. 7 (4), 17-29.
Conflicto de Intereses

Los autores declaran no tener ningún conflicto de intereses

Recibido: Febrero 18 de 2017 Aceptado: Marzo 31 de 2017 